\title{
On the Inflammation Theory of Cancer
}

\author{
Alain L Fymat* \\ International Institute of Medicine and Science, USA
}

Submission: December 01, 2017; Published: December 19, 2017

"Correspondence Address: Alain L Fymat, International Institute of Medicine and Science, USA, Tel: 760-485-9149;

Email: alain.fymat@fiimas.org

\section{Abstract}

A recent clinical trial on humans (CANTOS) aimed to test inflammation, one of the two major theories for cardiovascular diseases, the other theory being concerned with lipids. However, somewhat surprisingly, the trial provided some support for the inflammation theory of cancer and this result may overshadow the trial's original aim. However, inflammation has long been linked to cancer. As a preface to the link between inflammation and cancer, I first review the trial and summarize its results within the context of secondary prevention and mechanism pathways. With different doses against placebo, the drug tested in the trial was Canakinumab, a monoclonal antibody that targets Interleukin$1 \beta$. From a personalized medicine's perspective, the trial and its results point to the future of secondary prevention as the identification of which mechanistic pathway would be most beneficial for a given patient. From this viewpoint, inflammation today is very much analogous to the identification of cholesterol 30 years ago. We now need to distinguish those heart disease patients who have "residual cholesterol risk" from those who have "residual inflammatory risk", or both. These two groups will require different, perhaps also simultaneous, interventions.

Now, many malignancies arise in areas of chronic inflammation and inadequate resolution of inflammation could have a major role in tumor invasion, progression, and metastasis although it may not play a role in oncogenesis. Inflammation is of particular path physiological relevance in lung cancer in that chronic bronchitis, triggered by asbestos, silica, smoking, and other external inhaled toxins, results in a persistent inflammatory response. To interpret the trial results in terms of cancer, inflammation in the tumor microenvironment mediated by IL-1 $\beta$ was hypothesized to have a major role in cancer invasiveness, progression, and metastasis. This, in turn, suggests that IL-1 $\beta$ participates in the invasiveness of already existing malignancies. Thus, inhibition of IL- $1 \beta$ might have an adjunctive role in the treatment of cancers that have at least a partial inflammatory basis. This is the first evidence that inhibition of IL-1 $\beta$ with the monoclonal antibody Canakinumab is associated with reduced incidences of fatal cancer, lung cancer, and fatal lung cancer. The trial also showed an apparent decrease in the risk of cancer. Unfortunately, it was also associated with a higher incidence of fatal infection. Thus, anti-inflammatory therapy with Canakinumab targeting the IL-1 $\beta$ innate immunity pathway could significantly reduce incident lung cancer and lung cancer mortality. Nonetheless, these results should be replicated and extended in formal settings of cancer screening and treatment. They should also be directly related to early cancer screening and initial treatment, particularly in lung cancer.

Keywords: Anticoagulants; Antiplatelets; Cancer (Lung); Cardiovascular Diseases; Cytokines; Infection; Inflammation; Myocardial Infarction; Stroke; Thrombosis

Abbreviations: CANTOS: Canakinumab Anti-inflammatory Thrombosis Outcomes Study; CAD: Coronary Artery Disease; hs-CRP: High Sensitivity C-Reactive Protein; CV: Cardiovascular; CVD: CV Diseases; FI: Fatal Infection; HR: Hazard Ratio; IL: Inter-Leukin (1 $\beta, 6) ;$ IR: Incidence Rate; LDL: Low Density Lipoprotein; MI: Myocardial Infarction; NDA: New Frug Application; NHLBI: (U.S.) National Heart, Lung and Blood Institute; NLRP3: Nod-like Receptor Protein 3; PEE: Primary Efficacy Endpoint; SJIA: Systemic Juvenile Idiopathic Arthritis

\section{Disorders Mentioned}

Atherosclerosis; Basal Cell Carcinoma; Bypass Surgery; Cardiovascular Diseases; Cancer; Chronic Bronchitis; Chronic Fibrosis; Coronary Artery Disease; Cryopirin-Associated Periodic Syndrome; Gout; HIV; Hyperglyceridemia; Infection; Inflammation; Lung Adenocarcinoma; Lung Cancer; Melanoma; Myocardial Infarction; Osteoarthritis; Rheumatoid Arthritis; Sepsis; Squamous Cell Carcinoma; Stroke; Systemic Juvenile Idiopathic Arthritis; Thrombosis; Tuberculosis

\section{Drugs Listed}

Anacetrapib; ANGPTL3 Inhibitors; APOC3 Inhibitors; Aspirin; Beta-blocker; ACE-i (angiotensin converting enzyme); Canakinumab (Ilaris; a monoclonal antibody that targets interleukin-1ß); Clopidogrel; Colchicine; Ezetimibe; Gout; Lipoprotein(a) [Lp(a) Inhibitors]; Metformin; Methotrexate; Nod-Like Receptor Protein 3; PCSK9; Rivaroxaban; SGLT2 Inhibitors; Statin (reduce inflammation and recommended for high CRP and low LDL, angioplasty, and heart failure \}; Ticagrelor 


\section{Introduction}

In an earlier article [1], I reviewed the long quest for cancer cures for approximately the past 4,000 years. Since the time of Virchow [1,2], inflammation had been linked to cancer. In a subsequent article [3], I categorized the various theories (or hypotheses) of cancer advanced so far into exogenous and endogenous theories (eight in the former case and three in the latter, Table 1. At first hand, it might seem curious that

Table 1: Exogenous and endogenous theories of cancer. a recent clinical trial that tested the inflammation theory in cardiovascular diseases would provide some support for this theory in the case of cancer [4]. To understand this connection, one must hypothesize post facto that Inflammation in the tumor microenvironment has a major role in cancer invasiveness, progression, and metastasis. To begin with, it would be helpful to review briefly the Canakinumab Anti-inflammatory Thrombosis Outcomes Study (CANTOS) trial. Canakinumab is a human monoclonal antibody that targets interleukin-1 $\beta$ (IL-1 $\beta$ ).

\begin{tabular}{|c|c|}
\hline Exogenous & Endogenous \\
\hline Infectious blood suppuration & Endogenous virus \\
\hline Somatic mutation and exposure to environmental carcinogens & Two-hit - Proto-oncogenes and tumor suppressor genes \\
\hline Bacterial toxins & \\
\hline Infectious mononucleosis & \\
\hline Anti-vitamins & \\
\hline Anti-folates & \\
\hline Sensitivity to cytotoxic drugs & \\
\hline Inflammation & \\
\hline
\end{tabular}

\section{The Cantos Trial}

\section{Trial description and Results}

The CANTOS trial (funded by Novartis) was a double-blind, placebo-controlled trial conducted in 39 countries. It was aimed at investigating the role of inflammation in heart disease. It randomized 10,061 patients with a previous myocardial infarction (MI) and levels of high sensitivity C-reactive protein (hs-CRP, a measure of inflammation) above $2 \mathrm{mg} / \mathrm{L}$ as against four benchmarks:

i. Placebo,

ii. $50 \mathrm{mg}$ of Canakinumab iii. $150 \mathrm{mg}$ of Canakinumab, and

iv. $300 \mathrm{mg}$ of Canakinumab.

The drug was administered subcutaneously every three months. Canakitumab targets IL- $1 \beta$ innate immunity pathway; it is currently approved as an orphan drug for treatment of two rare pediatric conditions - systemic juvenile idiopathic arthritis (SJIA) and cryopyrin-associated periodic syndromes (CAPS) for which it is administered monthly. Trial results for CV events are summarized in Table 2 for placebo and the three treatment groups as a function of lipid level, high-sensitivity C-reactive protein (hs-CRP), primary efficacy endpoint (PEE, defined as non-fatal MI, non-fatal stroke, and CV death), incidence rates (per 100 persons/year), and rate of fatal infection.

Table 2: The CANTOS trial results for cardiovascular diseases.

\begin{tabular}{|c|c|c|c|c|c|}
\hline Treatment Group & Lipid Level & hs-CRP & Primary efficacy endpoint (PEE*) & $\begin{array}{c}\text { Incidence } \\
\text { Rates } \\
\text { (per 100 } \\
\text { Persons/yr) }\end{array}$ & $\begin{array}{c}\text { Rate of fatal } \\
\text { Infection }\end{array}$ \\
\hline Placebo & $\begin{array}{c}\text { No differences } \\
\text { between groups }\end{array}$ & & & 4.50 & 0.18 \\
\hline $50 \mathrm{mb}$ Canakinumab & $\begin{array}{c}\text { No differences } \\
\text { between groups }\end{array}$ & & Numerical reductions & 4.11 & 0.31 \\
\hline $150 \mathrm{mb}$ Canakinumab & $\begin{array}{l}\text { No differences } \\
\text { between groups }\end{array}$ & $37 \%$ reduction & \multirow{2}{*}{$\begin{array}{l}\text { Numerical reductions. Statistical } \\
\text { significance attributed to non-fatal } \\
\text { MI }+15 \% \text { reduction in primary } \\
\text { endpoint }+30 \% \text { reduction in need } \\
\text { for revascularization. No reduction in } \\
\text { mortality but a } 30 \% \text { reduction in need for } \\
\text { bypass surgery) }\end{array}$} & 3.86 & 0.28 \\
\hline $300 \mathrm{mb}$ Canakinumab & $\begin{array}{l}\text { No differences } \\
\text { between groups }\end{array}$ & $39 \%$ reduction & & 3.90 & 0.34 \\
\hline
\end{tabular}


After a median follow-up of 3.7 years, all three Canakinumab doses led to numerical reductions in the primary efficacy endpoint [nonfatal MI, nonfatal stroke, or cardiovascular (CV) death], but the 150-mg group was the only one that achieved statistical significance. Most of the difference was attributed to a reduction in the nonfatal MI endpoint. In the combined $150 / 300-m g$ treatment group, there was a $15 \%$ reduction in the primary endpoint and a $30 \%$ reduction in the need for revascularization procedures. The incidence rates (IR: events per 100 person-years) for the primary endpoint in each group were: Placebo: 4.50; $50 \mathrm{mg}$ : 4.11; $150 \mathrm{mg}$ : 3.86; and $300 \mathrm{mg}$ : 3.90. The corresponding fatal infection (FI) rates were: 0.18 , $0.31,0.28$, and 0.34 . There were no significant differences in all causes or CV mortality. However, the direction of the numbers favored Canakinumab. There is also what appears to be a real but small reduction in the primary endpoint $(2 \%$ absolute risk reduction) over the course of the trial.

Regarding the CV mortality benefit, there was a $30 \%$ reduction in the need for bypass surgery. Interestingly, the treatment had no effect on lipids, which suggests that the benefit was all attributable to the anti-inflammatory activity. The inflammatory hypothesis in no way competes with the lipid hypothesis. This result is biologically very interesting; especially with the lack of effect on lipids or other CV risk markers. However, it is a small effect and it comes with some real safety concerns.

Currently approved for use in rare inflammatory disorders, Canakinumab will cost $\$ 200,000$ per year in the U.S. Such pricing may perhaps be suitable for rare diseases, but not for a common indication such as coronary artery disease (CAD), even if given every 3 months. Less expensive anti-inflammatory drugs may one day be available for a CV inflammation indication. Thus, other trials are being tested for this purpose such as the National Heart, Lung and Blood Institute (NHLBI)-CIRT trial (cohort of 7,000 patients) by investigating Methotrexate, and the Lodoco trial from Australia and the Netherlands (cohort 4,300 patients), which is testing low dose Colchicine. It is worth noting that inflammation is an integral part of many salutary biological processes, especially the response to injury including infection, so some side effects related to infection are expected, but here the risk was serious relative to the benefits.

\section{Praises and Cautionary Remarks}

Broad arrays of cardiologists have praised the CANTOS trial and its results, the main praises being along the following lines:

a) "CANTOS is a remarkable scientific success, validating the concept that treatment of inflammation will reduce atherosclerosis. The discussion will now shift to risk-benefit and cost-benefit for this specific medicine."

b) "It shows for the first time that a drug that specifically targets inflammation and does not alter major serum lipids can reduce CVD events. Although we have pathological, physiological, and animal studies supporting the role of inflammation in atherosclerosis, this type of direct intervention shows we can manipulate the process and that specific targeting of inflammation may be a useful treatment."

c) "We are now entering an era with a plethora of treatment options for the secondary prevention of atherosclerotic CV disease. Human genetics has highlighted that this disease is multi-factorial, with several underlying causal pathways. We now have a treatment for several of these pathways."

d) "This study opened up many promising pathways."

e) "Although real, the modest results will have an outsized effect on the field."

f) "Allegedly, we now have evidence that manipulating the immune system can reduce CV risk. There are other targets for this drug, and there is the prospect that older drugs, such as Methotrexate, may produce marked benefit."

g) "The study has indicated that inflammation as a modifiable risk factor may be moving mainstream. Its results may have moved the inflammatory hypothesis of CAD forward scientifically and addressed the impact of modulating inflammatory cytokines in high-risk CV patients with elevated CRP."

However, other cardiologists recommend using caution for reasons such as the following:

i. "The concept that the first biologic tried for a complex disease like atherosclerosis would magically work seems like a long shot."

ii. "The likelihood of having an immediate effect on clinical practice is small and remote."

iii. "The cost of treatment cannot be justified in routine use in patients with previous MI until we understand more about the efficacy and safety trade-offs and unless a price restructuring and formal cost-effectiveness evaluation supports it."

iv. "Canakinumab will not become a major treatment since the effect size was small and there are several safety concerns."

v. "From a regulatory perspective, the treatment effect is not statistically persuasive (for a single trial); in fact the P-value for the 150-mg dose barely made it, which means that only a few extra events in the treatment arm would overturn statistical significance."

vi. "The treatment may not be allowed a claim for $\mathrm{CV}$ protection unless the sponsor submits a new drug application (NDA) for this indication."

vii. "The fatal infection risk will complicate the benefit-risk profile of this drug." 
Noting that those patients who had the largest CRP reduction had a large $25 \%-30 \%$ reduction in MI, the strategy was proposed in which patients with high levels of CRP might receive the first dose for free. Only those who experienced a large reduction in CRP would then go on to receive subsequent doses (and, of course, then pay the cost of the drug).

\section{Secondary Prevention and Mechanism Pathways}

After a heart attack, the average patient is now treated with several (generic) standard-of-care medications: Aspirin, statin, beta-blocker, ACE-i, and Metformin. Which additional medications should be added on? The options now include treatment for several distinct mechanisms:

a. LDL (PCSK9i, Ezetimibe, Anacetrapib).

b. Inflammation (Canakinumab).

c. Thrombosis/platelets (Ticagrelor, Clopidogrel).

d. Thrombosis/coagulation (low-dose Rivaroxaban).

e. Metabolic (SGLT2 Inhibitors).

f. Statins (reduce inflammation and recommended for high CRP and low LDL, angioplasty, and heart failure - all of which means a significant improvement in quality of life). Treatment was also associated with a reduction in gout, rheumatoid arthritis, and osteoarthritis.

And in the future, we will likely have therapies for:

i. Hypertriglyceridemia (APOC3 Inhibitors, ANGPTL3 Inhibitors).

ii. Lipoprotein (a) [Lp (a) Inhibitors].

How is the practicing physician to decide which therapies to add for any given patient? Indeed, the future of secondary prevention will be identifying which mechanistic pathway would be most beneficial for a given patient. Cardiologists will need to learn about inflammation today, the same way they learned about cholesterol 30 years ago. CANTOS is a demonstration of how personalized medicine will occur in the future, as we now need to distinguish those heart disease patients who have "residual cholesterol risk" from those who have "residual inflammatory risk", or both. These two groups will require different, perhaps also simultaneous, interventions.

\section{The Inflammation Theory of Cancer}

Many malignancies arise in areas of chronic inflammation $[5,6]$ and inadequate resolution of inflammation could have a major role in tumor invasion, progression, and metastasis $[7,8,9]$. Inflammation is of particular path physiological relevance in lung cancer in that chronic bronchitis, triggered by asbestos, silica, smoking, and other external inhaled toxins, results in a persistent inflammatory response [7,8]. Inflammatory activation in the lung is partly mediated through activation of the Nod-like receptor protein 3 (NLRP3) inflammasome, with consequent local generation of the active interleukin IL-1 $\beta$, a process that can lead to both chronic fibrosis and cancer $[9,10]$. In mice, inflammasome activation and pro-IL-1 $\beta$ processing accelerates tumor invasiveness, growth, and metastatic spread. For example [4], in IL-1 $\beta$ in mice, neither local tumors nor lung metastases developed after localized or intravenous inoculation with melanoma cell lines, which suggests that IL-1 $\beta$ participates in the invasiveness of already existing malignancies [10]. Thus, inhibition of IL-1 $\beta$ might have an adjunctive role in the treatment of cancers that have at least a partial inflammatory basis [11-15].

Inflammation in the tumor microenvironment mediated by $\mathrm{IL}-1 \beta$ is hypothesized to have a major role in cancer invasiveness, progression, and metastasis. An additional analysis [16] in CANTOS was undertaken with the aim of establishing whether inhibition of a major product of NLRP3 inflammasome with Canakinumab might alter cancer incidence. Two additional inclusion criterion were therefore added to the CANTOS inclusion criteria, namely that the patients were free of previously diagnosed cancer and, further, had concentrations of hs-CRP of $2 \mathrm{mg} / \mathrm{L}$ or greater. Participants were followed up for incident cancer diagnoses, which were adjudicated by an oncology endpoint committee that was masked to drug or dose allocation. Analysis was by intention to treat.

People with a history of chronic or recurrent infections, previous malignancy other than basal cell skin carcinoma, a suspected or known immune compromised state, or a history of (or at high risk for) tuberculosis or HIV-related disease, and those using systemic anti-inflammatory treatments were excluded.

\section{Cancer Results for Canakinumab}

While the CANTOS trial was not designed to explore its benefits for cancer, the fact that a mortality benefit from cancer was observed may well overshadow the more modest $\mathrm{CV}$ results.

Findings of the trial were as follows:

a. Baseline concentrations of hs-CRP (median $6 \bullet 0 \mathrm{mg} / \mathrm{L}$ vs $4 \bullet 2 \mathrm{mg} / \mathrm{L} ; \mathrm{p}<0 \bullet 0001)$ and IL-6 (3•2 vs $2 \bullet 6 \mathrm{ng} / \mathrm{L} ; \mathrm{p}<0 \bullet 0001)$ were significantly higher among participants subsequently diagnosed with lung cancer than among those not diagnosed with cancer.

b. During median follow-up of $3 \bullet 7$ years, compared with placebo, Canakinumab was associated with dose-dependent reductions in concentrations of hs-CRP of 26-41\% and of IL- 6 of $25-43 \%$ ( $p<0 \bullet 0001$ for all comparisons).

c. Total cancer mortality $(n=196)$ was significantly lower in the pooled Canakinumab group than in the placebo group ( $\mathrm{p}=0 \bullet 0007$ for trend across groups), but was significantly lower than placebo only in the $300 \mathrm{mg}$ group individually (hazard ratio [HR] 0•49 [95\% CI 0•31-0•75]; p=0•0009).

d. Incident lung cancer $(n=129)$ was significantly less frequent in the $150 \mathrm{mg}(\mathrm{HR} 0 \bullet 61$ [95\% CI 0•39-0•97]; 
$\mathrm{p}=0 \bullet 034)$ and $300 \mathrm{mg}$ groups (HR 0•33 [95\% CI 0•18-0•59]; $\mathrm{p}<0 \bullet 0001 ; \mathrm{p}<0 \bullet 0001$ for trend across groups).

e. Lung cancer mortality was significantly less common in the Canakinumab $300 \mathrm{mg}$ group than in the placebo group (HR 0•23 [95\% CI 0•10-0•54]; p=0•0002) and in the pooled Canakinumab population than in the placebo group ( $\mathrm{p}=0 \bullet 0002$ for trend across groups).

f. Fatal infections or sepsis were significantly more common in the Canakinumab groups than in the placebo group.

g. All-cause mortality did not differ significantly between the Canakinumab and placebo groups (HR 0•94 [95\% CI $0 \bullet 83-1 \bullet 06] ; p=0 \bullet 31$ ).

The trial provided the first evidence from a randomized trial in human beings that inhibition of IL- $1 \beta$ with the monoclonal antibody Canakinumab is associated with reduced incidences of fatal cancer, lung cancer, and fatal lung cancer. However, since the primary aim of the trial was to investigate cardiac events rather than cancer events, its exploratory data should be so interpreted, replicated, and extended in settings directly related to early cancer screening and initial treatment of cancers, particularly lung cancer. The cancer results diminish the impact of the CV outcome (which was paltry to begin with) but the clear dose response in the cancer signal and none in the $\mathrm{CV}$ endpoints is troublesome. These results are summarized in (Table 3).

Over the course of the study, there were 196 cancer deaths. Compared to placebo, there was a $14 \%$ reduction in the $50-\mathrm{mg}$ group, a $22 \%$ reduction with $150 \mathrm{mg}$, and a $51 \%$ reduction in the 300-mg group. The reduction in the 300-mg group achieved statistical significance, though the authors cautioned about the use of statistical tests in an exploratory analysis.

Nearly all the benefit occurred in lung cancer. Incident lung cancer was reduced by $23 \%$ in the 50 -mg group, $39 \%$ in the 150 mg group, and $67 \%$ in the 300-mg group. Fatal lung cancer was reduced by $77 \%$ in the $300-\mathrm{mg}$ group. However, Canakinumab may not have prevented cancer from starting. A more biologically plausible explanation is that Canakinumab reduced the rate of progression, invasiveness, and metastatic spread of lung cancers that were prevalent but undiagnosed at trial entry.

\section{Cancer Benefits}

The following cancer benefits accrued during the CANTOS trial (Table 3)

Table 3: Cancer results of the CANTOS trial.

\begin{tabular}{|c|c|c|c|c|}
\hline Treatment group & Cancer reduction & $\begin{array}{c}\text { Incident lung cancer } \\
\text { reduction }\end{array}$ & $\begin{array}{c}\text { Fatal lung cancer } \\
\text { reduction }\end{array}$ & $\begin{array}{c}\text { Incidence of fatal } \\
\text { infection }\end{array}$ \\
\hline Placebo & Baseline & Baseline & & 0.18 \\
\hline $50 \mathrm{mb}$ Canakinumab & $14 \%$ & $23 \%$ & & 0.32 \\
\hline $150 \mathrm{mb}$ Canakinumab & $22 \%$ & $39 \%$ & & \\
\hline $300 \mathrm{mb}$ Canakinumab & $51 \%$ & $67 \%$ & $77 \%$ & \\
\hline
\end{tabular}

a. Apparent decrease in risk of cancer.

b. Total cancer mortality, incident and fatal lung cancer reductions were lowest in the $300 \mathrm{mg}$ group.

Unfortunately, Canakinumab was associated with a higher incidence of fatal infection than placebo (rate was 0.18 in the 3,344 patient placebo group versus 0.32 among the 6,717 patients who received any dose of the drug), which worked out to 23 deaths versus 78 deaths $(\mathrm{P}=0.02)$. There was no significant difference in all-cause mortality. Nonetheless, the researchers' hypothesis-generating data suggest the possibility that anti-inflammatory therapy with Canakinumab targeting the IL-1 $\beta$ innate immunity pathway could significantly reduce incident lung cancer and lung cancer mortality. Nonetheless, the exploratory cancer data of CANTOS should be replicated and extended in formal settings of cancer screening and treatment. They should be directly related to early cancer screening and initial treatment of cancers, particularly lung cancer.

\section{Trial Follow-up}

During the median 3.7 years of trial follow-up, compared with placebo: a) Canakinumab was associated with dose-dependent reductions in hs-CRP of $26-41 \%$ ( $\mathrm{p}<0 \bullet 0001$ for all groups) and IL- 6 of $25-43 \%$ ( $<<0 \bullet 0001$ for all groups);

b) Canakinumab had no effect on LDL or HDL cholesterol;

c) Total cancer mortality was lower in the combined Canakinumab groups than in the placebo group.

d) The incidence rate of both cancer mortality and incidence rate of lung cancer per 100 person-years was increasingly lower for increasing Canakinumab dose groups compared with placebo.

e) Lung cancer accounted for $26 \%$ of all cancers and $47 \%$ of all cancer deaths in the placebo group, but only $16 \%$ of all cancers and $34 \%$ of cancer deaths in the Canakinumab groups.

f) The incidences of lung cancer of unspecified type, histologically defined lung adenocarcinoma, and histologically defined, poorly differentiated large cell cancer were significantly lower in the combined Canukinumab group than in the placebo group. 
g) There were too few cases for meaningful assessment of the effects of Canakinumab on the incidence of small-cell lung cancers or squamous cell carcinomas.

h) Compared with placebo, Canakinumab was not associated with significant reductions in incident cancers at other sites. The incidence of basal cell carcinoma per 100 person-years was higher in combined Canakinumab groups than in the placebo group, but this difference was not significant.

Further, stratification by smoking was affected. It suggested that the effect of Canakinumab on lung cancer was slightly stronger in current than in past smokers and was more prominent in the $300 \mathrm{mg}$ group.

\section{Summary and conclusions}

The CANTOS trial was designed to test the inflammation theory in cardiovascular diseases. It was surprising therefore that it would provide some support for this theory in the case of cancer. To understand this connection, one must hypothesize post facto that inflammation in the tumor microenvironment has a major role in cancer invasiveness, progression, and metastasis. To better understand this connection and establish a baseline, the trial was described and reviewed, and its results set forth. One must further remember that the trial subjects consisted of patients with increased hs-CRP concentrations who had had myocardial infarction, and in whom the rates of current or past smoking were high, that is patients at higher-than-average risk of lung cancer, While the trial was widely praised, several notes of caution ensued.

The trial benefits in the case of cancer may actually overshadow those for cardiovascular diseases. These exploratory data suggest that inhibition of IL- $1 \beta$ with Canakinumab over a median period of $3 \bullet 7$ years was associated with a reduction in the occurrence of fatal and non-fatal lung cancers among patients with atherosclerosis who had increased hs-CRP concentrations and who did not have a previous diagnosis of cancer. The effects were dose-dependent with relative hazard reductions of $67 \%$ for total lung cancer and $77 \%$ for fatal lung cancer in participants allocated to the Canakinumab $300 \mathrm{mg}$ dose (the largest dose tested). Patients with increased concentrations of the inflammatory biomarkers hs-CRP and IL- 6 had the highest risk for incident lung cancer. Smokers and those who achieved the greatest reductions in hs-CRP or IL- 6 seemed to gain the most benefit. By contrast, Canakinumab did not significantly affect the frequency of site-specific cancers other than lung cancer. However, total cancer mortality was more than $50 \%$ lower in the Canakinumab $300 \mathrm{mg}$ group than in the placebo group.

While this possibility cannot be ruled out, Canakinumab seems unlikely to have had direct effects on oncogenesis and the development of new lung cancers. A biologically plausible explanation is that Canakinumab reduced the rate of progression, invasiveness, and metastatic spread of lung cancers that were prevalent but undiagnosed at trial entry. This is in accord with previous experimental work that suggested that IL-1 $\beta$ can promote angiogenesis and tumor growth and that it is essential to tumor invasiveness in already existing malignant cells. The data also suggest that Canakinumab should be assessed as a potential therapy for early lung cancers (or after or in combination with other well-known test procedures), lung cancer prevention, and treatment based on genetic screening within loci associated with inflammatory cytokine function.

The major toxicity of Canakinumab in CANTOS was a significant increase in fatal infection and sepsis. This adverse effect was balanced, however, by the reduction in cancer mortality such that no increase in non-cardiovascular or allcause mortality was noted.

\section{References}

1. Fymat AL (2016) The long quest for cancer cures. J Cancer Prev Curr Res 6(2): 1-3.

2. Balkwill F, Mantovani A (2001) Inflammation and cancer: Back to Virchow? Lancet 357(9255): 539-545.

3. Fymat AL (2017) On cancer's theories, true nature, and possible selferadicating process. Can Ther Oncol Int J 7(2): 1-3.

4. Ridker PM (2017) The CANTOS trial". Presented at the 2017 European Society of Cardiology meeting in Barcelona. New England Journal of Medicine, UK.

5. Coussens M, Werb Z (2002) Inflammation and cancer. Nature 420(6917): 860-867.

6. Grivennikov SI, Greten FR, Karin M (2010) Immunity, inflammation, and cancer. Cell 140(6): 883-899.

7. Apte RN, Dotan S, Elkabets M (2006) The involvement of IL-1 in tumorigenesis, tumor invasiveness, metastasis and tumor-host interactions. Cancer Metastasis Rev 25(3): 387-408.

8. Porta C, Larghi P, Rimoldi M (2009) Cellular and molecular pathways linking inflammation and cancer. Immunobiology 214(9-10): 761-777.

9. Balkwill FR, Mantovani A (2012) Cancer-related inflammation: Common themes and therapeutic opportunities. Semin Cancer Biol 22(1): $33-40$

10. O Callaghan DS, O Donnell, DO Connell F, O Byrne KJ (2010) The role of inflammation in the pathogenesis of non-small cell lung cancer. J Thorac Oncol 5(12): 2024-2036.

11. Lee JM, Yanagawa J, Peebles KA, Sharma S, Mao JT (2008) Inflammation in lung carcinogenesis: New targets for lung cancer chemoprevention and treatment. Crit Rev Oncol Hematol 6: 208-217.

12. Dinarello CA, Simon A, van der, Meer JW (2012) Treating inflammation by blocking interleukin- 1 in a broad spectrum of diseases. Nat Rev Drug Discov 11(8): 633-652.

13. Dinarello CA (2010) Why not treat human cancer with interleukin-1 blockade? Cancer Metastasis Rev 29(2): 317-329.

14. Apte RN, Voronov E (2008) Is interleukin-1 a good or bad "guy"in tumor immunobiology and immunotherapy? Immunol Rev 222: 222241.

15. Ridker PM, Mac Fadyen JG, Thuren T, Everett BM, Libby P, et al (2017) Effect of interleukin-1 $\beta$ inhibition with Canakinumab on incident lung cancer in patients with atherosclerosis: Exploratory results from a randomized, double-blind, placebo-controlled trial. The Lancet, Uk. 
16. Lewis AM, Varghese $\mathrm{S}, \mathrm{Xu} \mathrm{H}$, Alexander HR (2006) Interleukin-1 and cancer progression: The emerging role of interleukin-1 receptor

This work is licensed under Creative

Commons Attribution 4.0 License

DOI: $10.19080 /$ CTOIJ.2017.08.555740 antagonist as a novel therapeutic agent in cancer treatment. J Transl Med 4: 48.

Your next submission with Juniper Publishers will reach you the below assets

- Quality Editorial service

- Swift Peer Review

- Reprints availability

- E-prints Service

- Manuscript Podcast for convenient understanding

- Global attainment for your research

- Manuscript accessibility in different formats ( Pdf, E-pub, Full Text, Audio)

- Unceasing customer service

Track the below URL for one-step submission https://juniperpublishers.com/online-submission.php 